\title{
Circularly Polarized Huygens Source Antenna Based on Two Stacked Dielectric Resonators
}

\author{
C. D. Morales*†, J. B. de Araújo*†, C. Morlaas*, A. Chabory*, R. Pascaud ${ }^{\dagger}$, M. Grzeskowiak ${ }^{\dagger}$, G. Mazingue ${ }^{\ddagger}$ \\ *ENAC, Université de Toulouse, France, email: carlosdavid.moralespena@enac.fr \\ $\dagger$ ISAE-SUPAERO, Université de Toulouse, France \\ $\ddagger$ Anywaves, Toulouse, France
}

\begin{abstract}
In this paper, a cross-shaped dielectric resonator antenna arranged in a stacked configuration is proposed to realize a Huygens source in circular polarization at 2.45 GHz ISM band. The antenna principle relies on the combination of two pairs of orthogonal electric and magnetic dipoles. The antenna is designed and optimized with numerical analysis using far-field simulations in Ansys HFSS. It finally has a radiation pattern oriented in one half-space without the use of a ground plane.
\end{abstract}

Index Terms-Dielectric resonator antenna, Huygens source, circular polarization, half-space radiation.

\section{INTRODUCTION}

The interest in small-size autonomous platforms is growing rapidly. UAVs are, for instance, more and more used in the fields of media, agriculture, and industry. Another example concerns the space industry where nanosatellites could yield a significant reduction in the launching and operation costs. These two applications share the common constraint of embedding complex systems without a large metallic plate for antenna support.

This integration constraint is important for antenna design in terms of size, polarization and radiation pattern. Such pattern requires improved radiation properties in the forward direction and low backward radiation despite the lack of a ground plane. These requirements can be satisfied by designing a Huygens source, i.e., an antenna with a half-space radiation pattern.

Based on works related to magneto-electrical sources to obtain circular polarization (CP) [1], and a Huygens source radiation pattern [2], some antennas with cardioid radiation pattern and $\mathrm{CP}$ properties have been proposed [3][4][5].

In a previous paper [6], the authors have presented a single-fed linearly polarized Huygens source antenna based on stacked dielectric resonators (DRs). As a new contribution, we present an original solution to design a Huygens source in CP. It consists of a cross-shaped antenna made of two stacked rectangular DRs with different electrical and physical properties.

\section{ANTENNA PRINCIPLE AND DESIGN}

\section{A. Antenna principle}

The antenna principle to design a Huygens source in $\mathrm{CP}$ relies on the orthogonal combination of two linearly polarized Huygens sources. According to the coordinate system shown in Fig. 1. the first Huygens source which is linearly polarized along the $x z$-plane, is due to the $\mathrm{TE}_{111}^{x}$ and quasi- $\mathrm{TM}_{111}^{y}$ modes in the DR, which radiate a magnetic and electric radiation pattern-like, respectively [7]. The quasi- $\mathrm{TM}_{111}^{y}$ is denoted here in reference to the corresponding cylindrical resonator mode. On the other hand, the second Huygens source which is linearly polarized along the $y z$-plane, is generated by the $\mathrm{TE}_{111}^{y}$ and quasi-TM $\mathrm{TM}_{111}^{x}$ modes in the DR.

\section{B. Dielectric resonator antenna design}

The dielectric resonator antenna (DRA) at $f_{0}=2.45 \mathrm{GHz}$ ISM band is designed to realize a Huygens source pattern, both theoretically using the dielectric waveguide model (DWM) [7] and numerically using eigen mode analysis in Ansys HFSS. The cross-shaped antenna is made up of two stacked DRs, as shown in Fig. 1. The stacked cross-shaped DR is fed using two coaxial probes $90^{\circ}$ out of phase to excite the $\mathrm{TE}_{111}^{x}$ and $\mathrm{TE}_{111}^{y}$ modes with the required phase difference in order to achieve CP. In addition to that, the quasi-TM $\mathrm{TM}_{111}^{x}$ and quasi$\mathrm{TM}_{111}^{y}$ modes are excited using the electric field distribution of the $x$-directed and $y$-directed magnetic dipoles, respectively. Here, a proper frequency shift between TE and quasi-TM modes allows to satisfy the requirements to obtain $\mathrm{CP}$. This frequency shift is accomplished by setting the electrical and physical properties of the stacked DR.

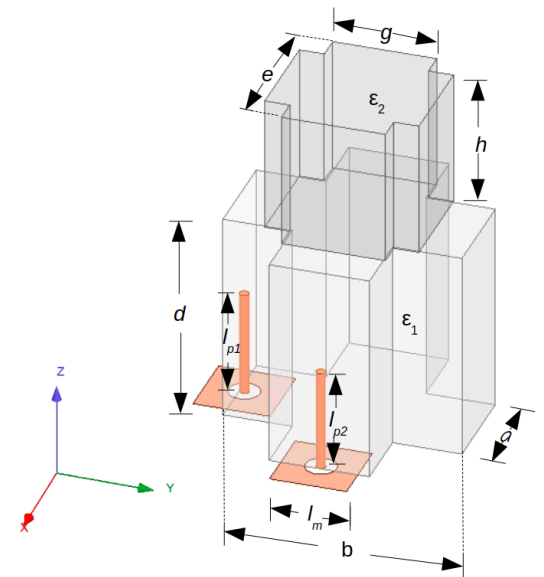

Fig. 1: Stacked cross-shaped DRA.

A parametric analysis of the antenna dimensions is performed in order to adjust the frequency shift between TE and 
quasi-TM modes. The electrical and physical properties of the DRs are $a=13 \mathrm{~mm}, b=d=31 \mathrm{~mm}, e=h=20$ $\mathrm{mm}, g=13.5 \mathrm{~mm}, \epsilon_{r 1}=11.5$, and $\epsilon_{r 2}=18$. Finally, the total electrical antenna dimensions are $d+h=0.41 \lambda_{0}$, and $b=0.25 \lambda_{0}$. The probes have a diameter of $d_{f}=1.23 \mathrm{~mm}$, and lengths of $l_{p 1}=15.5 \mathrm{~mm}$ and $l_{p 2}=15 \mathrm{~mm}$. The probes are supported by small metallic plates with square dimensions of $l_{m}=0.08 \lambda_{0}$ to ensure the impedance matching. Table II shows the eigen modes for the stacked cross-shaped DRA. As a result, we observe that the desired modes radiate around $f_{0}$.

TABLE I: Resonance frequency $(\mathrm{GHz})$ of the eigen modes in the optimized cross-shaped DR.

\begin{tabular}{|l|c|c|}
\hline Modes & $\begin{array}{c}\mathrm{TE}_{111}^{x} \\
\mathrm{TE}_{111}^{y}\end{array}$ & $\begin{array}{c}\mathrm{TM}_{111}^{x} \\
\mathrm{TM}_{111}^{y}\end{array}$ \\
\hline Resonance frequency & 2.35 & 2.72 \\
\hline
\end{tabular}

\section{Antenna Performances}

Fig 2 shows the simulated $S_{11}$ and $S_{21}$. The antenna has an impedance bandwidth of $21.2 \%$ from $2.26 \mathrm{GHz}$ to $2.78 \mathrm{GHz}$, for a reflection coefficient of $\left|S_{11}\right| \leq 10 \mathrm{~dB}$. Furthermore, a low coupling between the two coaxial probes ensures a good orthogonality between the desired TE and quasi-TM modes.

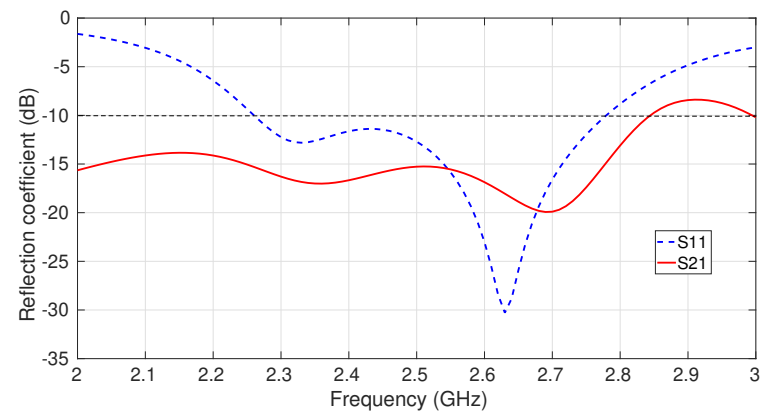

Fig. 2: Simulated reflection coefficient.

The total simulated front-to-back $(F / B)$ and axial ratio (AR) of the antenna are shown in Fig. 3 The Huygens source antenna achieves the largest $F / B$ ratio of $31.8 \mathrm{~dB}$ at $2.43 \mathrm{GHz}$. Besides, the DRA has a $F / B$ ratio bandwidth $(F / B \geq 15 \mathrm{~dB})$ of $5.3 \%$ from $2.38 \mathrm{GHz}$ to $2.51 \mathrm{GHz}$, and an AR bandwidth (AR $\leq 3 \mathrm{~dB}$ ) equal to $22.9 \%$ from $2.22 \mathrm{GHz}$ to $2.78 \mathrm{GHz}$.

Fig. 4 illustrates the simulated radiation patterns of the proposed antenna. It corresponds to a Huygens source. Furthermore, the DRA operates in left-hand $\mathrm{CP}$ at $2.45 \mathrm{GHz}$ with a maximum simulated gain at boresight of $5.93 \mathrm{dBi}$.

\section{CONCLUSION}

A stacked cross-shaped DRA has been designed in circular polarization in the $2.45 \mathrm{GHz}$ ISM band to achieve a Huygens source radiation pattern. The methodology relies on the combination of two orthogonal pairs of TE and quasi-TM modes.

The proposed DRA has an impedance bandwidth of $21.2 \%$, and a $3-\mathrm{dB}$ axial ratio bandwidth of $22.9 \%$. Furthermore, it operates in left-hand circular polarization with a maximum simulated gain of $5.93 \mathrm{dBi}$ at $2.45 \mathrm{GHz}$.

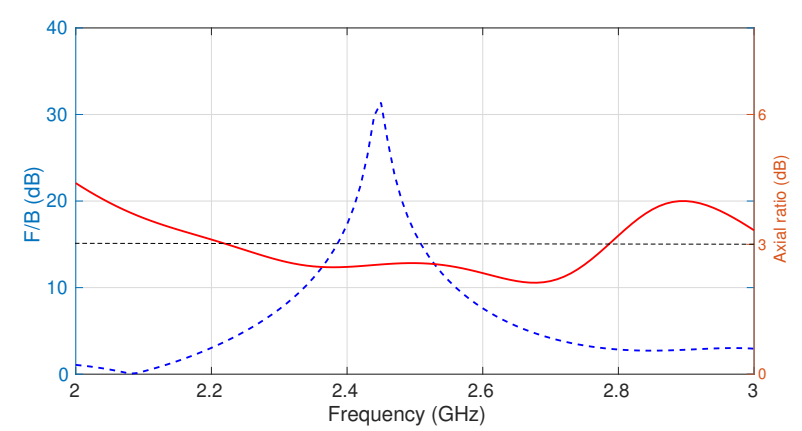

Fig. 3: Simulated front-to-back and axial ratios.

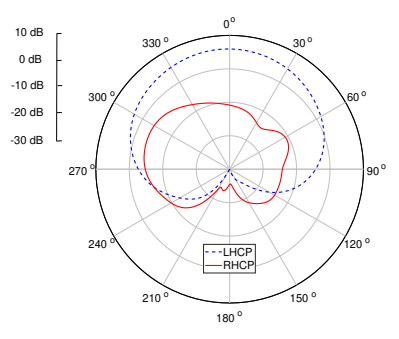

(a)

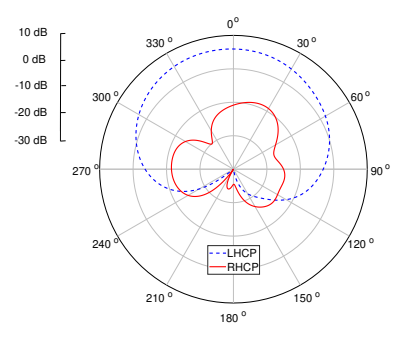

(b)
Fig. 4: Simulated total antenna gain $(\mathrm{dBi})$ at $2.45 \mathrm{GHz}$, considering $\phi=0^{\circ}$ and $\phi=90^{\circ}$.

Due to the need of a heterogeneous dielectric, additive manufacturing can be used to build the proposed antenna. Moreover, in order to manage the permittivity difference between the upper and lower DRs, a periodized structure of several unit cells using 3-D printing of ceramics can be envisaged, as previously done in [8].

\section{ACKNOWLEDGEMENT}

The authors acknowledge the Région Occitanie and ENAC for their financial support.

\section{REFERENCES}

[1] H. A. Wheeler, "A helical antenna for circular polarization," Proceedings of the IRE, vol. 35 , no. 12, pp. 1484-1488, 1947.

[2] A. Chlavin, "A new antenna feed having equal e-and h-plane patterns," Transactions of the IRE Professional Group on Antennas and Propagation, vol. 2, no. 3, pp. 113-119, 1954.

[3] P. Alitalo, A. Karilainen, T. Niemi, C. Simovski, and S. Tretyakov, "Design and realisation of an electrically small huygens source for circular polarisation," IET microwaves, antennas \& propagation, vol. 5, no. 7, pp. 783-789, 2011.

[4] M. Li and K.-M. Luk, "Wideband magnetoelectric dipole antennas with dual polarization and circular polarization," IEEE Antennas and Propagation Magazine, vol. 57, no. 1, pp. 110-119, 2015.

[5] C. Morlaas, B. Souny, and A. Chabory, "Helical-ring antenna for hemispherical radiation in circular polarization," IEEE Transactions on Antennas and Propagation, vol. 63, no. 11, pp. 4693-4701, Nov 2015.

[6] C. Morales, C. Morlaas, A. Chabory, R. Pascaud, M. Grzeskowiak, and G. Mazingue, "Huygens source antenna using stacked dielectric resonators," in 2020 IEEE International Symposium on Antennas and Propagation and North American Radio Science Meeting, 2020, pp. 1-2.

[7] A. Petosa, Dielectric resonator antenna handbook. Artech House Publishers, 2007.

[8] M. Thevenot, C. Menudier, N. Delhote, O. Tantot, M. Romier, and N. Capet, "A dielectric resonator antenna designed with a structured dielectric material," in 2018 IEEE Conference on Antenna Measurements \& Applications (CAMA). IEEE, 2018, pp. 1-2. 